\title{
Powers of Convex-Cyclic Operators
}

\author{
Fernando León-Saavedra ${ }^{1}$ and María del Pilar Romero-de la Rosa ${ }^{2}$ \\ ${ }^{1}$ Department of Mathematics, University of Cádiz, Campus de Jerez de la Frontera, Cádiz, 11405 Jerez de la Frontera, Spain \\ ${ }^{2}$ IES Sofía, Doctor Marañón 13, Cádiz, 11407 Jerez de la Frontera, Spain
}

Correspondence should be addressed to María del Pilar Romero-de la Rosa; pilar.romero@uca.es

Received 21 January 2014; Revised 15 April 2014; Accepted 16 April 2014; Published 8 May 2014

Academic Editor: Manuel Maestre

Copyright ( 2014 F. León-Saavedra and M. P. Romero-de la Rosa. This is an open access article distributed under the Creative Commons Attribution License, which permits unrestricted use, distribution, and reproduction in any medium, provided the original work is properly cited.

\begin{abstract}
A bounded operator $T$ on a Banach space $X$ is convex cyclic if there exists a vector $x$ such that the convex hull generated by the orbit $\left\{T^{n} x\right\}_{n \geq 0}$ is dense in $X$. In this note we study some questions concerned with convex-cyclic operators. We provide an example of a convex-cyclic operator $T$ such that the power $T^{n}$ fails to be convex cyclic. Using this result we solve three questions posed by Rezaei (2013).
\end{abstract}

\section{Introduction and Main Results}

Throughout this paper we denote by $L(X)$ the algebra of all bounded linear operators on a real or complex infinite dimensional Banach space $X$. An operator $T \in L(X)$ is said to be cyclic if there exists a vector $x \in X$ (later called cyclic vector for $T$ ) such that the linear span of the orbit

$$
\text { linear } \operatorname{span}\left(\left\{T^{n} x: n \in \mathbb{N}\right\}\right)
$$

is dense in $X$. If the orbit $\operatorname{Orb}(T, x):=\left\{T^{n} x: n \in \mathbb{N}\right\}$ is dense itself, without the help of the linear span, then $T$ is called hypercyclic and $x$ is called hypercyclic for $T$. In the midway stand several notions studied by different authors. For instance, the operator $T$ is said to be supercyclic if the projective orbit is dense in $X$. We refer to the books $[1,2]$ and references therein for further information on hypercyclic operators.

When we sometimes abusively say that a polynomial $p(z)$ is a convex polynomial, what we really mean is that $p(z)=t_{0}+$ $t_{1} z+t_{2} z^{2}+\cdots+t_{n} z^{n}, t_{i} \in \mathbb{R}, i=0, \ldots, n$, and $t_{0}+t_{1}+\cdots+t_{n}=1$. We will focus our attention on the notion of convex cyclicity introduced by Rezaei in [3]. An operator $T$ is said to be convex cyclic if there exists a vector $x \in X$ such that the real convex hull of the orbit (denoted by $\operatorname{co}(\operatorname{Orb}(T, x)))$

$$
\operatorname{co}(\operatorname{Orb}(T, x))=\{p(T) x: p \text { convex polynomial }\}
$$

is dense in $X$.
In [3] are characterized the convex-cyclic matrices in finite dimension, and the author develops the main properties in the infinite dimensional setting.

A result by Ansari [4] states that if $T$ is a hypercyclic operator then $T^{n}$ is also hypercyclic; this fact is not true for cyclic operators. In this paper we show that Ansari's result fails also for convex-cyclic operators, solving a question posed in [3].

Another result proved by Bourdon and Feldman on hypercyclic operators says that if the orbit of a vector is somewhere dense, then it is dense (see [5]). From our previous counterexample we can construct a non-convexcyclic operator $T$ such that the $\operatorname{co}(\operatorname{Orb}(T, x))$ has nonempty interior. That is, Bourdon and Feldman's result is not true in the convex-cyclic setting. Finally we can construct a convexcyclic operator $T$ such that $T$ is not weakly hypercyclic; that is, its orbit is not dense in the weak operator topology. The later examples solve Questions 5.5 and 5.6 in [3].

\section{Powers of a Convex-Cyclic Operator}

The first example of hypercyclic operator on Banach spaces was discovered by Rolewicz (see [6]). Throughout this section $\mathscr{B}=\ell_{p} 1 \leq p<\infty$ or $c_{0}$ of complex valued sequences. Rolewicz's operator $\mu B$ with $|\mu|>1$ is defined on $\mathscr{B}$ by

$$
\mu B\left(x_{0}, x_{1}, \ldots, x_{n}, \ldots\right)=\mu\left(x_{1}, x_{2}, \ldots, x_{n}, \ldots\right),
$$

where $B$ denotes the backward shift operator. 
Lemma 1. Set $\alpha=e^{2 \pi i / 3}$ and $r_{0}>1$. For any $z_{0} \in \mathbb{C} \backslash\{0\}$ there exist $k_{0} \geq 0$ and a sequence of polynomials $p_{k}(z)$ such that

(1) $p_{k}(z)=\left(t_{1, k}+t_{2, k} z+t_{3, k} z^{2}\right) z^{k}$ for all $k \geq k_{0}$;

(2) $t_{i, k} \in[0,1]$ and $t_{1, k}+t_{2, k}+t_{3, k}=1, i \in\{1,2,3\}$ and $k \geq k_{0}$

(3) $p_{k}\left(r_{0} \alpha\right)=z_{0}, k \geq k_{0}$.

Proof. Let us denote by $\mathscr{T}$ the triangle with vertices $\left\{1, r_{0} \alpha, r_{0}^{2} \alpha^{2}\right\}$. Since $\left|r_{0} \alpha\right|>1$ and $0 \in \mathscr{T}$, there exists $k_{0}$ such that $z_{k}=z_{0} /\left(r_{0} \alpha\right)^{k} \in \mathscr{T}$ for all $k \geq k_{0}$. Then, there exist barycentric coordinates $t_{i, k} \in[0,1] i=1,2,3$ satisfying $t_{1, k}+t_{2, k} r_{0} \alpha+t_{3, k}\left(r_{0} \alpha\right)^{2}=z_{k}$ and $t_{1, k}+t_{2, k}+t_{3, k}=1$. Then, the polynomials $p_{k}(z)=\left(t_{1, k}+t_{2, k} z+t_{3, k} z^{3}\right) z^{k}$, for all $k \geq k_{0}$, yield the desired result.

Lemma 2. Let $p_{k}$ be a sequence of polynomials satisfying Conditions (1)-(3) of Lemma 1. Then, there exists a $G_{\delta}$ dense subset $Z \subset \mathscr{B}$ of vectors such that $\left\{p_{k}(\mu B) x_{0}\right\}_{k \geq k_{0}}$ is dense in $\mathscr{B}$ for all $x_{0} \in Z$.

Proof. We will use some hypercyclicity criterion version for sequence of operators (see [2, Theorem 3.24]); that is, we will show the existence of two dense subsets $X$ and $Y$ and a sequence of mappings $S_{k}$ such that

(i) $\lim _{k} p_{k}(\mu B) x=0 \forall x \in X$;

(ii) $p_{k}(\mu B) S_{k} y=y \forall y \in Y$;

(iii) $\lim _{k} S_{k} y=0 \forall y \in Y$.

Let us consider the subsets

$$
\begin{gathered}
X=\operatorname{span}\{\operatorname{Ker}(\mu B-\lambda I):|\lambda|<1\}, \\
Y=\{\operatorname{Ker}(\mu B-\lambda I): \lambda \in \mathbb{R}, 1<\lambda<|\mu|\},
\end{gathered}
$$

which are dense in $\mathscr{B}$ (see [2, Example 3.2, page 70]).

If $x \in \operatorname{Ker}(\mu B-\lambda I)$ with $|\lambda|<1$, then

$$
\begin{aligned}
p_{k}(\mu B) x & =\left(t_{1, k}(\mu B)^{k}+t_{2, k}(\mu B)^{k+1}+t_{3, k}(\mu B)^{k+2}\right) x \\
& =\left(t_{1, k} I+t_{2, k}(\mu B)+t_{3, k}(\mu B)^{2}\right)(\mu B)^{k} x \\
& \leq \text { const }|\lambda|^{k},
\end{aligned}
$$

which goes to zero when $k \rightarrow \infty$; therefore, Condition (i) is fulfilled.

Denoting by $q_{k}(z)=t_{1, k}+t_{2, k} z+t_{3, k} z^{2}$, since $t_{1, k}, t_{2, k}$, $t_{3, k}$ are barycentric coordinates of a triangle, then $q_{k}(\lambda)$ lies in the degenerate triangle with vertices $\left\{1, \lambda, \lambda^{2}\right\}$, in particular $q_{k}(\lambda) \geq 1$.

Let us take $y \in \operatorname{Ker}(\mu B-\lambda I)$ with $\lambda \in \mathbb{R}$ and $1<\lambda<|\mu|$, and let us define the mapping $S_{k}$ on $y$ as

$$
S_{k} y=\frac{1}{\lambda^{k} q_{k}(\lambda)} y
$$

and we extend linearly $S_{k}$ on $Y$. Clearly $S_{k} y \rightarrow 0$ as $k \rightarrow \infty$ for all $y \in Y$ and $p_{k}(\mu B) S_{k} y=y$ for all $y \in Y$. Thus, by the hypercyclicity criterion there exists a $G_{\delta}$ dense subset of vectors $x_{0} \in \mathscr{B}$ such that $\left\{p_{k}(\mu B) x_{0}\right\}_{k \geq k_{0}}$ is dense in $\mathscr{B}$.
Now, let us prove the main result of this section, which solves Question 5.6 in [3].

Theorem 3. The operator $T=r_{0} \alpha I_{\mathbb{C}} \oplus \mu B$ is convex cyclic on $\mathbb{C} \oplus \mathscr{B}$; however $T^{3}$ is not.

Proof. If $p$ is a polynomial, then $p(T)=p\left(r_{0} \alpha\right) \oplus p(\mu B)$. Let us observe that the first coordinate of the powers of $\left(T^{3}\right)^{n}$ are only real numbers. Take $x=\sum_{n=0}^{\infty} x_{0} e_{0} \in \mathbb{C} \oplus \mathscr{B}$. If $f^{\star}$ is the projection on the first coordinate,

$$
\left\{f^{\star}\left(\operatorname{co}\left(\operatorname{Orb}\left(T^{3}, x\right)\right)\right)=t x_{0}, \forall t \geq r_{0}\right\}
$$

which is not dense in $\mathbb{C}$. Therefore, $T^{3}$ is not convex cyclic.

Now, let us prove that $T$ is a convex-cyclic operator using a direct application of the Baire category theorem (see, for instance, [2, Theorem 1.57]). Thus $T$ is convex cyclic if for any nonempty open subsets $U, V \subset \mathbb{C} \oplus \mathscr{B}$, there exists a convex polynomial $p(z)$ such that $p(T)(U) \cap V \neq \emptyset$.

Indeed, let $U=G_{1} \times W_{1}$ and $V=G_{2} \times W_{2}$ open subsets of $\mathbb{C} \oplus \mathscr{B}$, where $G_{i} \subset \mathbb{C}$ and $W_{i} \subset \mathscr{B}, i=1,2$, are nonempty open subsets. Let $z_{1} \in G_{1}$ and $z_{2} \in G_{2}$ with $z_{1} z_{2} \neq 0$. Set $z_{0}=z_{2} / z_{1}$ and $p_{k}(z)$ the sequence of polynomials which guarantees Lemma 1. Hence we have $p_{k}\left(r_{0} \alpha\right)=z_{2} / z_{1}$ and therefore $p_{k}\left(r_{0} \alpha\right) z_{1}=z_{2}$ (this fact will imply that $p_{k}(T)$ acting on $G_{1}$ will intersect $G_{2}$ ). Now we apply Lemma 2 and we obtain a $G_{\delta}$ dense subset $Z \subset \mathscr{B}$ of hypercyclic vectors for the sequence $\left\{p_{k}(\mu B)\right\}$. Thus there exist $x_{0} \in W_{1}$ and a subsequence $\left\{n_{k}\right\}$ such that $p_{n_{k}}(\mu B) x_{0} \in W_{2}$. Therefore $p_{n_{k}}(T)(U) \cap V \neq \emptyset$, which yields the desired result.

Remark 4. If we take $\alpha=e^{2 \pi i / n}$ with $n \geq 4$, using similar arguments as in Theorem 3, we can show that $T=r_{0} \alpha \oplus \mu B$ is convex cyclic on $\mathbb{C} \oplus \mathscr{B}$ but $T^{n}$ is not (if $n=4$ the operator $T$ is convex cyclic but $T^{2}$ is not).

Remark 5. If we consider the Rolewicz operator on real spaces $\ell^{p}, 1 \leq p<\infty$ or $c_{0}$, then we can get that the operator $T=-r_{0} \oplus \mu B\left(r_{0}>1\right)$ is convex cyclic on $\mathbb{R} \oplus \ell^{p} 1 \leq p<\infty$ or $\mathbb{R} \oplus c_{0}$, but clearly $T^{2}$ is not. Lemma 1 can be adapted clearly to the real case. Now it is well known that if we consider Rolewicz's operator on real spaces $\ell^{p}, 1 \leq p<\infty$ or $c_{0}$, then its complexification can be identified with the same operator on the corresponding spaces of complex sequences. With some slight modification in the proof of Corollary 2.51 in [2] we can obtain that Lemma 2 continues being true on real spaces. The rest of the proof is straightforward.

Remark 6. Another difference between hypercyclic operators and convex-cyclic operators is the following: hypercyclic operators are invariant under unimodular multiplications (see [7]); that is, if $T$ is hypercyclic, then $\lambda T$ is also with $|\lambda|=1$. However this is not true for convex-cyclic operators; the previous counterexample $T=r_{0} \alpha I_{\mathbb{C}} \oplus \mu B$ is convex cyclic; however $\bar{\alpha} T$ is not.

Now, let $\alpha=e^{2 \pi i / 3}$. Let us consider the operator $T=$ $\alpha I_{\mathbb{C}} \oplus \mu B$, that is, the same operator provided by Theorem 3 but without the multiplier factor $r_{0}$. Then, an easy check shows 
that the set $S=\left\{p_{k}(\alpha): p_{k}(z)\right.$ convex polynomial $\}$ is contained in the unit disk. Moreover, the set $S$ has nonempty interior in $\mathbb{C}$; for instance, $S$ contains the triangle $\mathscr{T}$ with vertices $\left\{1, \alpha, \alpha^{2}\right\}$. Using the arguments of Theorem 3 we can find a vector $x_{0} \in \mathscr{B}$ such that the convex orbit

$$
\left\{p(T)\left(1 \oplus x_{0}\right): p \text { convex polynomial }\right\}
$$

is dense in $\mathscr{T} \oplus \mathscr{B}$. Therefore the convex orbit has nonempty interior. However the operator $T$ is not convex cyclic. This solves Question 5.5 in Rezaei's paper.

Proposition 7. Bourdon and Feldman's result fails for convexcyclic operators.

The adjoint of the operator $T$ in Theorem 3 has an eigenvalue; therefore, $T$ cannot be weakly hypercyclic. This solves Question 5.4 in [3].

\section{Conflict of Interests}

The authors declare that there is no conflict of interests regarding the publication of this paper.

\section{Acknowledgments}

The authors are indebted to the referees for alerting them to some details that they had to take care of in the proof of the first version. Fernando León-Saavedra and María del Pilar Romero de la Rosa were partially supported by Junta de Andalucía FQM-257.

\section{References}

[1] F. Bayart and E. Matheron, Dynamics of Linear Operators, vol. 179 of Cambridge Tracts in Mathematics, Cambridge University Press, Cambridge, UK, 2009.

[2] K.-G. Grosse-Erdmann and A. P. Manguillot, Linear Chaos. Universitext, Springer, London, UK, 2011.

[3] H. Rezaei, "On the convex hull generated by orbit of operators," Linear Algebra and Its Applications, vol. 438, no. 11, pp. 41904203, 2013.

[4] S. I. Ansari, "Hypercyclic and cyclic vectors," Journal of Functional Analysis, vol. 128, no. 2, pp. 374-383, 1995.

[5] P. S. Bourdon and N. S. Feldman, "Somewhere dense orbits are everywhere dense," Indiana University Mathematics Journal, vol. 52, no. 3, pp. 811-819, 2003.

[6] S. Rolewicz, "On orbits of elements," Studia Mathematica, vol. 32, pp. 17-22, 1969.

[7] F. León-Saavedra and V. Müller, "Rotations of hypercyclic and supercyclic operators," Integral Equations and Operator Theory, vol. 50, no. 3, pp. 385-391, 2004. 


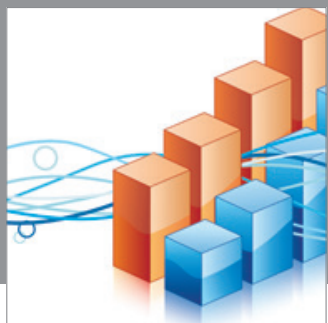

Advances in

Operations Research

mansans

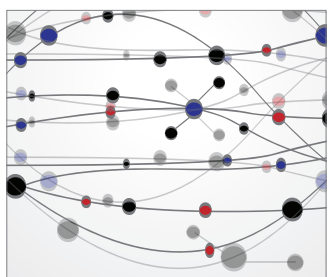

The Scientific World Journal
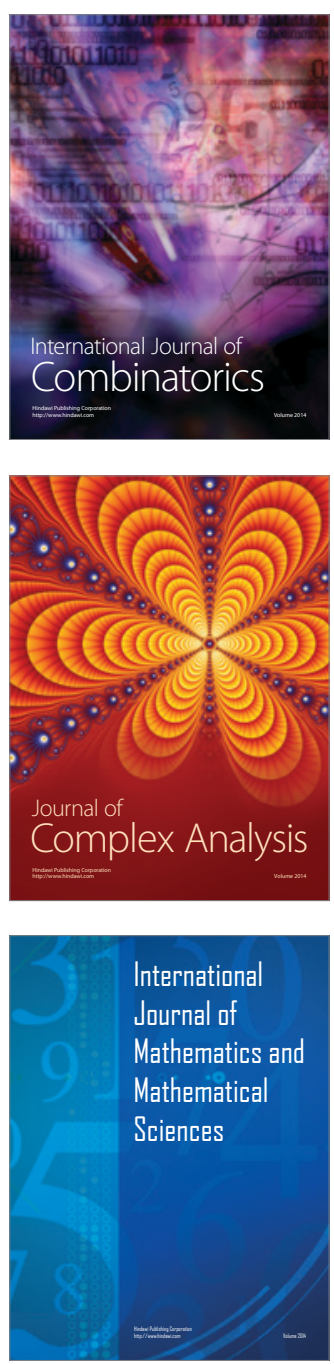
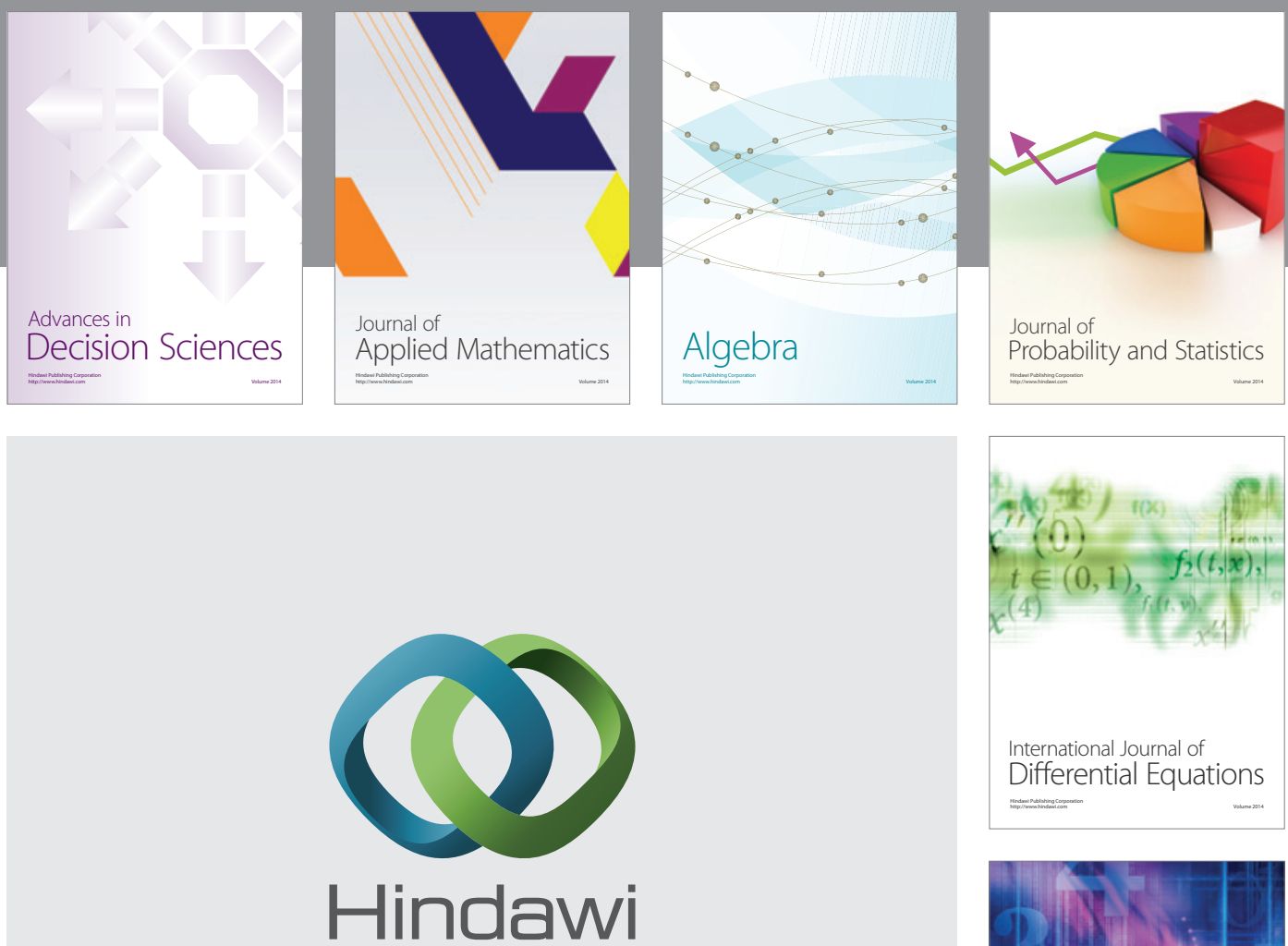

Submit your manuscripts at http://www.hindawi.com
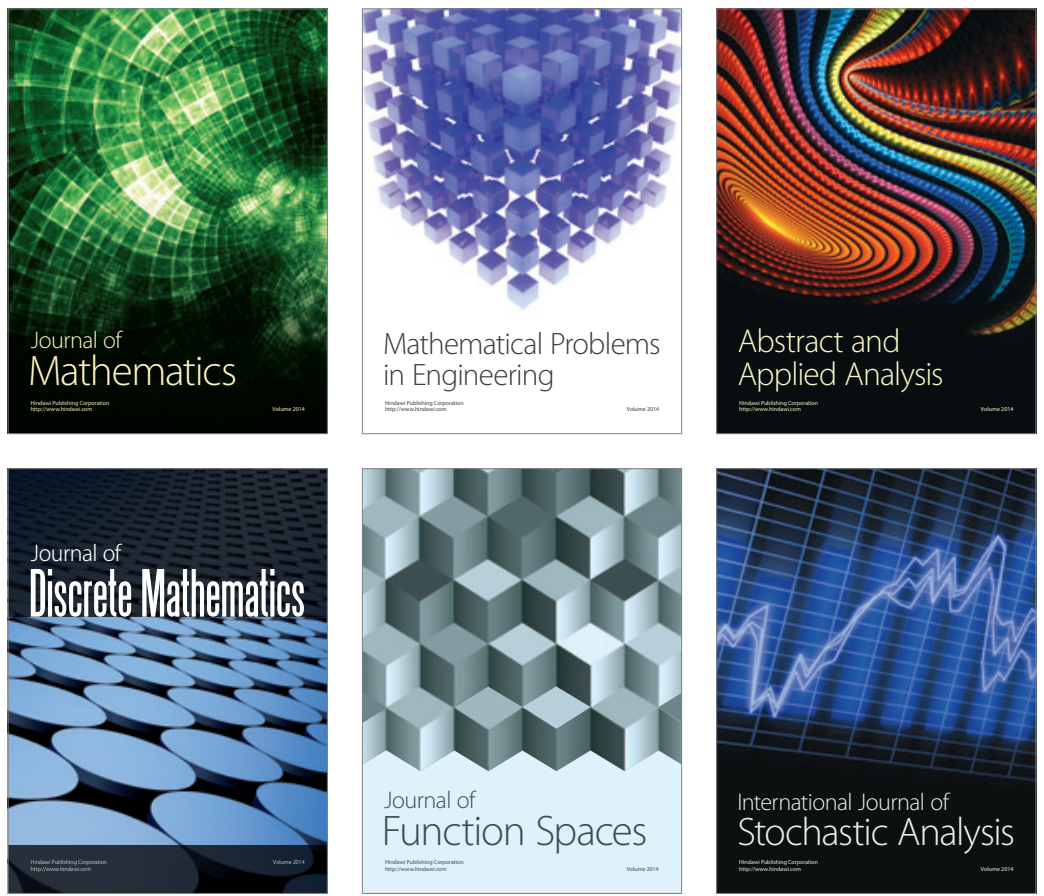

Journal of

Function Spaces

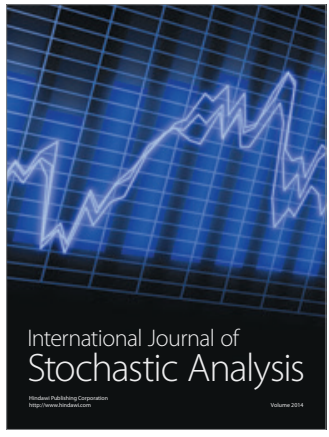

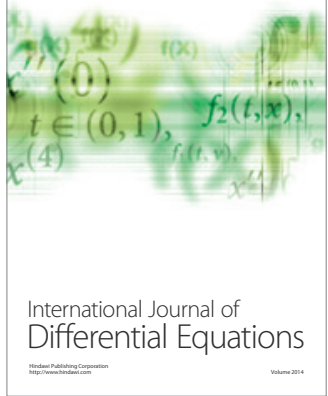
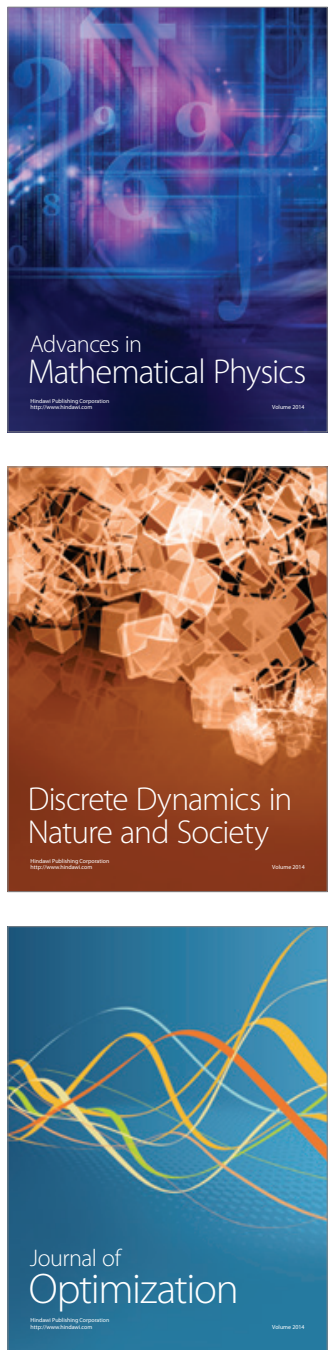